\title{
Fusion of cancer stem cells and mesenchymal stem cells contributes to glioma neovascularization
}

\author{
CHAO SUN, DONGLIANG ZHAO, XINGLIANG DAI, JINSHENG CHEN, XIAOCI RONG, HAIYANG WANG, \\ AIDONG WANG, MING LI, JUN DONG, QIANG HUANG and QING LAN
}

Department of Neurosurgery, The Second Affiliated Hospital of Soochow University, Suzhou, Jiangsu 215000, P.R. China

Received May 4, 2015; Accepted June 22, 2015

DOI: $10.3892 / o r .2015 .4135$

\begin{abstract}
The ability of tumor cells to autonomously generate tumor vessels has received considerable attention in recent years. However, the degree of autonomy is relative. Meanwhile, the effect of bone marrow-derived mesenchymal stem cells (BMSCs) on tumor neovascularization has not been fully elucidated. The present study aimed to illuminate whether cell fusion between glioma stem cells and BMSC is involved in glioma neovascularization. BMSCs were isolated from transgenic nude mice, of which all nucleated cells express green fluorescent protein (GFP). The immunophenotype and multilineage differentiation potential of BMSC were confirmed. SU3 glioma stem/progenitor cells were transfected with red fluorescent protein (SU3-RFP cells). In a co-culture system of BMSC-GFP and SU3-RFP, $\mathrm{RFP}^{+} / \mathrm{GFP}^{+}$cells were detected and isolated by dual colors using FACS. The angiogenic effect of $\mathrm{RFP}^{+} / \mathrm{GFP}^{+}$cells was determined in vivo and in vitro. Flow cytometry analysis showed that BMSC expressed high levels of CD105, C44, and very low levels of CD45 and CD11b. When co-cultured with SU3-RFP, 73.8\% of cells co-expressing RFP and GFP were identified as fused cells in the 5th generation. The fused cells exhibited tube formation ability in vitro and could give rise to a solid tumor and form tumor blood vessels in vivo. In the dual-color orthotopic model of transplantable xenograft glioma, yellow vessel-like structures that expressed CD105, RFP and GFP were identified as de novo-formed vessels derived from the fused cells. The yellow vessels observed in the tumor-bearing mice directly arose from the fusion of BMSCs and SU3-RFP cells. Thus, cell fusion is one of the driving factors for tumor neovascularization.
\end{abstract}

Correspondence to: Dr Qing Lan, Department of Neurosurgery, The Second Affiliated Hospital of Soochow University, Suzhou, Jiangsu 215000, P.R. China

E-mail: szlqsci@163.com

Key words: fluorescence tracing method, cell fusion, bone marrowderived mesenchymal stem cells, tumor neovascularization, CD105 protein

\section{Introduction}

The cellular and molecular mechanisms of tumor angiogenesis and its prospects for anti-angiogenic cancer therapy are major issues for both cancer biology and targeted cancer therapy. Multiple mechanisms of tumor neovascularization have been established, yet considerable controversy persists (1).

Among these mechanisms, angiogenesis and vasculogenesis are widely accepted. In the first mechanism, vascular endothelial cells of the host sprout from preexisting vasculature to form new vessels; in the second mechanism, bone marrow-derived endothelial progenitor cells are recruited to form new blood vessels (2). However, the contribution of these two mechanisms to the process of tumor neovascularization remains unclear.

Recent research has indicated that the tumor neovascularization process is more complex than previously assumed (3). Vasculogenic mimicry (VM) was first found in human melanoma by Maniotis et al in 1999 (4). Unlike the angiogenesis and vasculogenesis mechanisms, VM facilitates tumor cells to form functional blood vessels in the absence of endothelial cells as has been witnessed in numerous solid tumors such as breast, prostate, ovarian and lung cancer, synovial sarcoma, rhabdomyosarcoma, pheochromocytoma and glioma (5).

Meanwhile, the effect of tumor stem/progenitor cells on tumor neovascularization is attracting increased attention. Our research group (6,7), Ricci-Vitiani et al (8) and Wang et al (9) reported that glioma stem cells directly participate in the formation of tumor vessels by transdifferentiating or differentiating into endothelial cells. In addition, there is increasing evidence that mesenchymal stem cells (MSCs) have the ability to migrate to tumor sites and exert stimulatory or inhibitory effects on tumor angiogenesis through direct and/or indirect interaction with tumor cells (10).

The main divergence is whether tumor vascular cells are transformed from tumor cells or supplied by the host vascular cells. In the present study, human glioma stem/progenitor SU3 cells were transfected with red fluorescent protein (SU3-RFP cells), and then co-cultured with bone marrow-derived mesenchymal stem cells (BMSCs)-GFP, and fused cells co-expressing RFP and green fluorescent protein (GFP) were identified and detected for their tube formation ability in vitro and biological characteristics in vivo; meanwhile, SU3-RFP cells were inoculated into the brains of GFP 
nude mice. In the xenograft tumors, de novo tumor vessels that originated from the cell fusion of tumor cells and host BMSCs were detected.

\section{Materials and methods}

Cells and animals. The human glioma stem/progenitor cell line SU3 was previously established in our laboratory (11). According to the published methods by which we established SU1 and SU2 (12), SU3 was obtained from a surgical specimen of an adult male patient diagnosed with glioblastoma multiforme. SU3 cells expressed CD133 and nestin consistent with the characteristics of glioma stem cells (11). SU3 cells were transfected with the RFP gene using a lentiviral-mediated gene transfection kit (GeneChem, Shanghai, China). Under a fluorescence microscope (Zeiss Axio Observer A1; Carl Zeiss, Germany), nearly $100 \%$ of the tumor cells expressed RFP (13). SU3 cells with stable RFP expression were isolated using flow cytometry (FACSCanto II; BD Biosciences, USA) and were amplified.

NC-C57BL/6J-GFP nude mice (6-8 weeks of age) with whole-body expression of the GFP gene were prepared by our research group (14). Foxn $1^{\text {nu }}$ mice were purchased from the Model Animal Research Center of Nanjing University (Nanjing, China). The mice were housed under specific pathogen-free conditions with a 12-h light/dark cycle and controlled temperature at the Laboratory Animal Center of Soochow University.

\section{BMSC isolation and culture}

Isolation. NC-C57BL/6J-GFP nude mice (6-8 weeks of age) were sacrificed by $\mathrm{CO}_{2}$ asphyxiation. After being immersed in $75 \%$ alcohol for 5-10 $\mathrm{min}$, the mice were placed on a sterile culture dish. Both femurs and tibiae were dissected out, and bone marrow plugs were extracted by flushing the bone marrow cavity with phosphate-buffered saline (PBS) containing penicillin and streptomycin.

Culture. Bone marrow-derived cells were cultured in complete medium containing Dulbecco's modified Eagle's medium (DMEM; Gibco, USA) and 10\% fetal bovine serum (FBS; HyClone, USA). The cells were plated on a 24 -well plate ( $3 \times 10^{6}$ cells/well) and were incubated at $37^{\circ} \mathrm{C}$ in a humidified atmosphere with 5\% $\mathrm{CO}_{2}$. After 3-4 days, the adherent cells attained confluency, and the non-adherent cells were discarded. At this point, the cells were considered to be at stage 0 (P0). The confluent cells were detached with Accutase (Innovative Cell Technologies, San Diego, CA, USA) and passaged. The culture medium was replaced every 3 days.

\section{Biological characteristics of the BMSCs}

Phenotypic analysis by flow cytometry. BMSCs were harvested with Accutase and washed twice with PBS, and then $5 \times 10^{5}$ cells were suspended in $10 \mu \mathrm{l}$ of PBS for binding with each specific antibody. BMSCs were then incubated in the dark for $30 \mathrm{~min}$ at room temperature with antibodies against CD45, CD11b, CD44 and CD105 (Abcam, Cambridge, UK). Flow cytometry was performed on the FACSCanto II (BD Biosciences).

\section{Mesenchymal differentiation}

Adipogenesis differentiation. BMSCs were seeded at a density of $5 \times 10^{4}$ cells $/ \mathrm{ml}$ in a 6 -well plate, cell differentiation was induced with adipogenic differentiation medium which contained $10 \%$ FBS, $1 \mu \mathrm{M}$ dexamethasone (Sigma, USA), $500 \mu \mathrm{M}$ 1-methyl3-isobutyl xanthine (Sigma), $60 \mu \mathrm{M}$ indomethacin (Sigma) and $5 \mu \mathrm{M}$ insulin (Sigma). The medium was replaced every 3 days. After 2 weeks, adipocytes were visualized by Oil-O-Red staining for fatty drops.

Osteogenesis differentiation. BMSCs were incubated in DMEM/F12 supplemented with $10 \%$ FBS, $0.1 \mu \mathrm{M}$ dexamethasone, $10 \mathrm{mM} \beta$-glycerophosphate (Sigma), $50 \mu \mathrm{M}$ ascorbic acid-2-phosphate (Sigma). On day 21, cells were performed Alizarin Red S staining to detect osteogenesis.

Chondrogenic differentiation. BMSCs were cultured in high-glucose DMEM (Invitrogen) supplemented with 10\% FBS, $100 \mathrm{nM}$ dexamethasone, $1 \mathrm{mM}$ sodium pyruvate (Invitrogen), $10 \mu \mathrm{M}$ insulin, $50 \mathrm{mg} / \mathrm{l}$ ascorbate 2-phosphate, and $10 \mathrm{ng} / \mathrm{ml}$ transforming growth factor- $\beta$ (Sigma). On day 28 , cells were cultured on the coverslide and fixed with paraformaldehyde, then incubated with a rabbit polyclonal anti-mouse collagen II antibody (Abcam, ab34712, 1:200). The secondary antibody used was a peroxidase-conjugated anti-rabbit IgG (Vector Labs, MP7401). Positive reaction was defined as blue using DAB Peroxidase Substrate Kit (Blue Color, Boster, AR1025, Wuhan, China).

Colony forming unit assay. Colony forming cell assay was based on the method of Liu et al (15), BMSCs at P5 were seeded into 6-well plates at 10-100 cells/well in duplicates. Culture media were changed every 3 days. On day 10, the cells were stained with $0.25 \%$ crystal violet (Santa Cruz, Dallas, TX, USA) for $10 \mathrm{~min}$ and then observed under an inverted fluorescence microscope (Zeiss Axio Observer A1).

Co-culture of BMSCs and SU3-RFP cells. The SU3-RFP cells were added to the BMSCs at a ratio of $1: 15$. Half of the culture medium was renewed every 3 days, and the cells were passaged once they reached $80-90 \%$ confluency. Briefly, we removed and discarded the culture medium and rinsed the cell layer with $\mathrm{Ca}^{++} / \mathrm{Mg}^{++}$-free Dulbecco's PBS. We then added 2.0-3.0 $\mathrm{ml}$ of Accutase to the dishes. After $2 \mathrm{~min}$, we added 2.0-3.0 $\mathrm{ml}$ of complete culture medium and aspirated the cells by gentle pipetting. Finally, we centrifuged the mixture for $5 \mathrm{~min}(1,000 \mathrm{rps}, 179 \mathrm{x} \mathrm{g})$ and subcultivated the cells at a ratio of 1:2. Cell growth was observed under an inverted fluorescence microscope. $\mathrm{RFP}^{+} / \mathrm{GRP}^{+}$cells were detected by flow cytometry and sorted.

In vitro tube formation assay of the fused cells. To detect the tube formation ability of the fused cells, $100 \mu \mathrm{l}$ of Matrigel (BD Biosciences, San Jose, CA, USA) was poured onto a 24-well dish and placed in a $\mathrm{CO}_{2}$ incubator (Jouan, France; with $5 \% \mathrm{CO}_{2}$ at $\left.37^{\circ} \mathrm{C}\right)$. Fused cells $\left(\mathrm{RFP}^{+} / \mathrm{GFP}^{+}\right.$cells $)$in DMEM containing 10\% FBS supplemented with $5 \mathrm{ng} / \mathrm{ml}$ basic fibroblast growth factor (bFGF) and $10 \mathrm{ng} / \mathrm{ml}$ epidermal growth factor (EGF) (both from Gibco), were seeded onto each well at a density of $2 \times 10^{4}$ cells/well. The cells were periodically observed under an inverted microscope (Zeiss Axio Observer A1).

In vivo experiments. To investigate the ability of the fused cell to form endothelial vessels in vivo, $1 \times 10^{5}$ sorted $\mathrm{RFP}^{+} / \mathrm{GFP}^{+}$cells suspended in $20 \mu \mathrm{l}$ PBS were injected into the right caudate 
nucleus of Foxn $1^{\text {nu }}$ mice with the assistance of a stereotaxic apparatus. After 3-4 weeks, the mice were sacrificed and the xenograft tumors were harvested, and continuously sectioned at a thickness of $5 \mu \mathrm{m}$. The sections were either stained with hematoxylin and eosin (H\&E) or observed under a fluorescence microscope.

To establish the dual-color orthotopic model of transplantable xenograft glioma, $1 \times 10^{5} \mathrm{SU} 3-\mathrm{RFP}$ cells were injected into the right caudate nucleus of NC-C57BL/6J-GFP nude mice using a 20- $\mu 1$ Hamilton syringe with the assistance of a stereotaxic apparatus. All of the procedures were carried out under general anesthesia by intraperitoneal injection of $10 \%$ chloral hydrate $(200 \mathrm{mg} / \mathrm{kg})$. After 3-4 weeks, the tumor xenografts were sampled and cut into two pieces. One-half of the fresh tumor tissue was cut into $1 \mathrm{~mm}^{3}$ pieces and pressed on slides for fluorescence microscopy. The other tumor was embedded in the Optimal Cutting Temperature medium, frozen in liquid nitrogen. The frozen samples were continuously sectioned at a thickness of $5 \mu \mathrm{m}$. Nuclei were stained with DAPI, then either observed under a fluorescence microscope or performed immunohistochemical or H\&E staining.

Immunocytochemical/immunohistochemical staining. Immunocytochemical staining of CD105 was performed on SU3-RFP cells, BMSCs and fused cells, while immunohistochemical staining of CD105 and CD31 was performed on the tissue sections. Briefly, $5 \times 10^{3}$ cells were placed on a slide on a 24 -well plate, and the slide was taken out when covered by $80-90 \%$ of the cell population. Meanwhile, a frozen section of the xenograft tumor was made. Primary antibodies used were rabbit polyclonal antibody to CD105/endoglin (ab107595, dilution 1:250) and rabbit polyclonal antibody to CD31 (ab28364, dilution 1:50) (both from Abcam). After incubation with the primary antibodies at $4^{\circ} \mathrm{C}$ overnight, the slides were incubated with peroxidase-conjugated anti-rabbit IgG (MP7401) and stained with diaminobenzidine (DAB) chromogen solution (SK-4105) (both from Vector Laboratories), and then counterstained with hematoxylin.

Statement of ethics. The present study received approval from the Ethics Committee of the Second Affiliated Hospital of Soochow University. The animal experiments were approved by the Medical Review Board of Soochow University, and all procedures were conducted in accordance with the Chinese laws governing animal care.

Statistical analysis. Data are expressed as mean \pm SEM. Statistical significance was determined by the Student's t-test. A value of $\mathrm{P}<0.05$ was considered to indicate a statistically significant result.

\section{Results}

BMSC immunophenotype analysis by flow cytometry. We investigated the immunophenotypes of the BMSCs (passages 1, 3 and 5) using immunofluorescence flow cytometry. As shown in Fig. 1, BMSCs expressed high levels of CD105 and CD44, but very low levels of CD45 and CD11b.

Biological characteristics of the BMSCs. In this experiment, BMSCs were collected from the bone marrow of hybrid
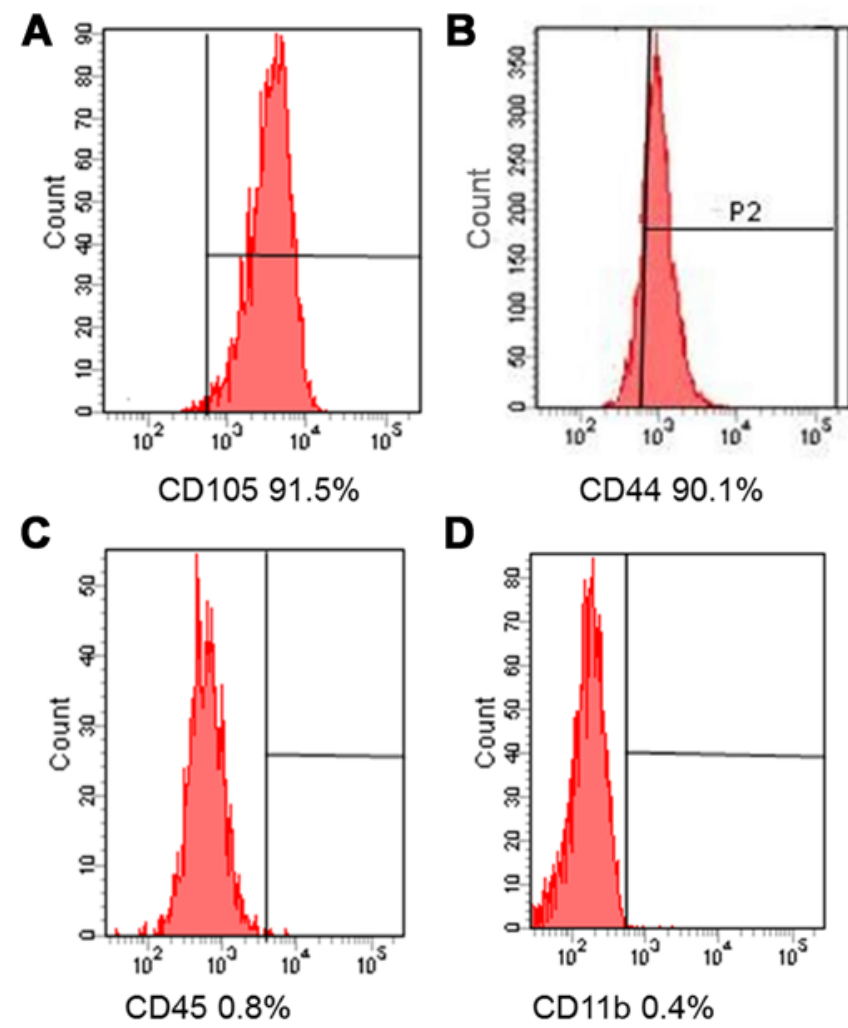

Figure 1. Analysis of bone marrow-derived mesenchymal stem cells for surface marker expression using flow cytometric analysis.

generations of NC nude and C57BL/6J-GFP mice. According to the specific experimental requirements, several passages were performed in vitro, and the biological characteristics of the cells were investigated. The results indicated that the 1st to 5th generation of BMSCs expressed GFP protein (Fig. 2A-C) and had the capacity to form cell colonies (Fig. 2C). Immunocytochemical staining of BMSCs at P1, P3 and P5 indicated that most of these cells expressed CD105 (Fig. 2D-F). To investigate the ability of the BMSCs to differentiate into osteoblasts, adipocytes, and chondroblasts in vitro, we induced cells of passages 1,3 , and 5 under different culture conditions. We found that all the samples from various donors kept their multipotent differentiation potential (Fig. 3).

All of the above results indicated that the BMSCs in this experiment had the following three major characteristics: i) they consisted of a major proportion of $\mathrm{CD} 105^{+}$cells; ii) they exhibited stable biological characteristics at passages 1-5; and iii) $100 \%$ of the BMSCs expressed GFP. Therefore, the BMSCs were suitable for co-culture with tumor cells and were used to study the effect of host BMSCs on tumor neovascularization based on fluorescence tracing.

Harvesting $\mathrm{RFP}^{+} / \mathrm{GFP}^{+}$cells from the co-culture of SU3-RFP cells and BMSCs. SU3-RFP cells were added to the BMSCs at the ratio of 1:15 and were then co-cultured. On day 4, we were able to detect a small proportion of GFP and RFP double-positive cells under an inverted fluorescence microscope (Fig. 4A-C), which were identified as $\mathrm{RFP}^{+} / \mathrm{GFP}^{+}$fused cells. With the extension of culture and passage, the proportion of fused cells increased. Detection by flow cytometry showed 

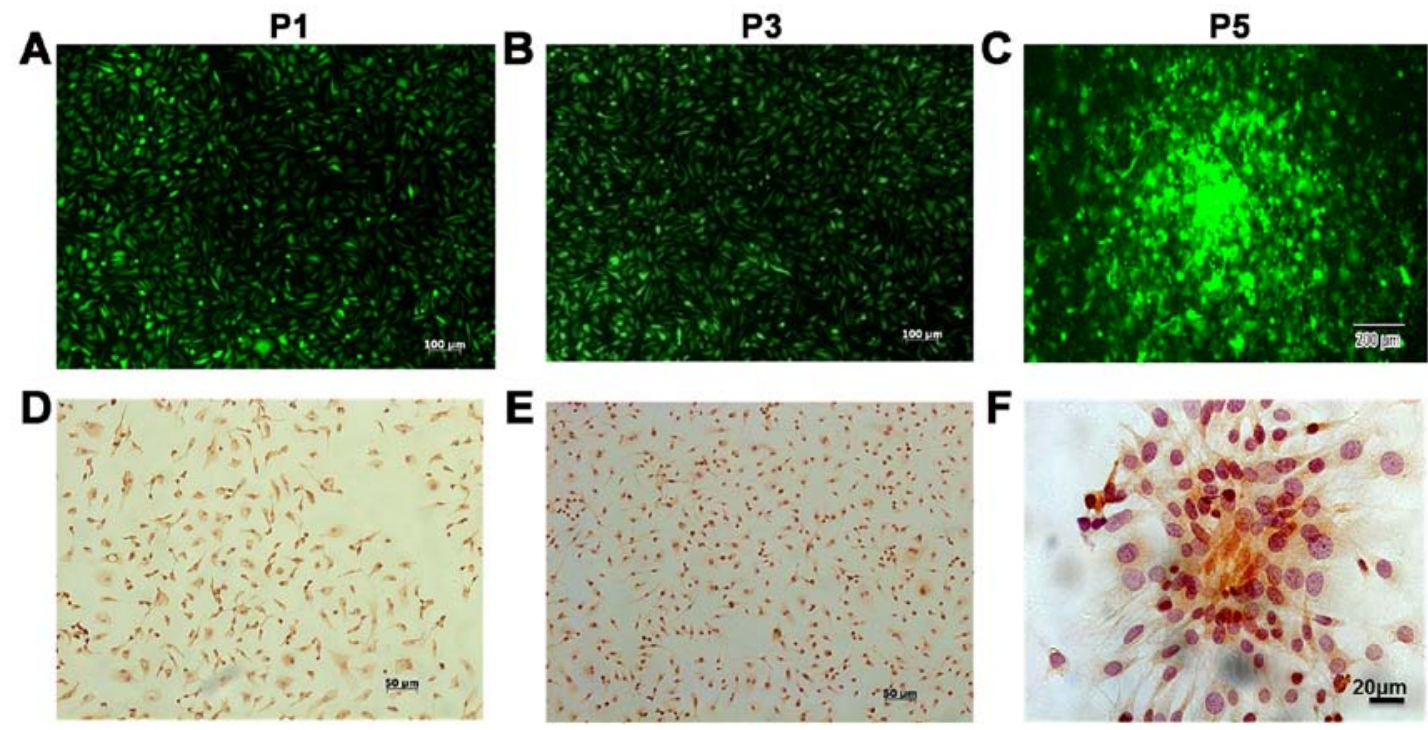

Figure 2. Characteristics and immunocytochemical staining of bone marrow-derived mesenchymal stem cells (BMSCs) cultured in vitro. Cells at (A) P1, (B) P3 and (C) P5 as observed by inverted fluorescence microscopy. Nearly 100\% of the BMSCs expressed GFP. (C) Cell colonies on culture at P5 under an inverted fluorescence microscope. (D-F) Immunocytochemical staining of CD105 in the BMSCs at (D) P1 and (E) P3. (F) Colonies showed that most of the cells expressed CD105. GFP, green fluorescent protein.

A

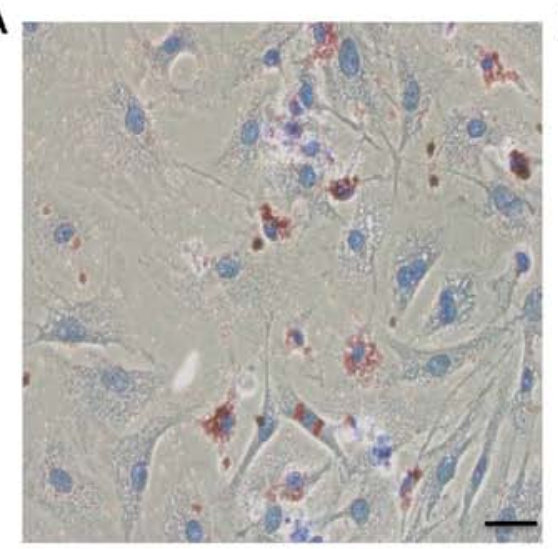

B

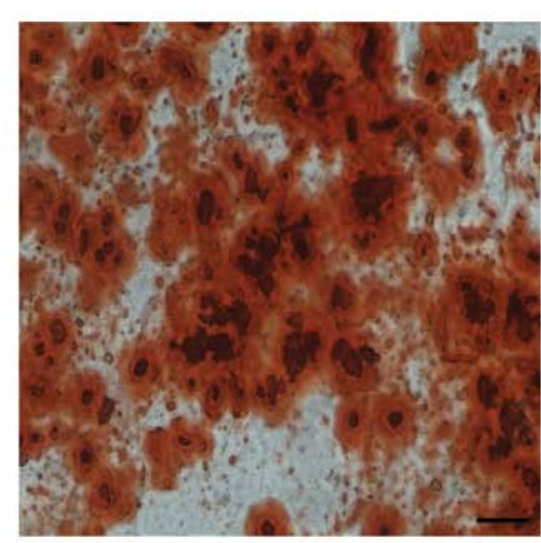

C

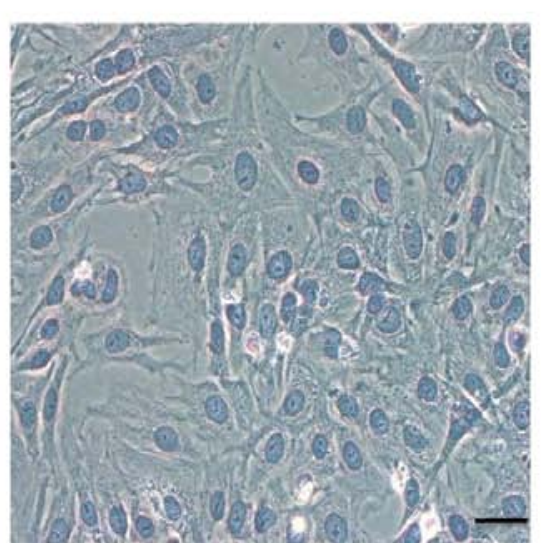

Figure 3. Trilineage differentiation of BMSC from a random donor at P3. (A) BMSC differentiation to adipocytes was shown by Oil-O-Red staining. (B) Alizarin Red S staining to detect osteogenesis differentiation. (C) Immunocytochemical staining showed expression of intracellular collagen II in chondrocytes (scale bars, $50 \mu \mathrm{m})$.

that the proportion of yellow $\mathrm{RFP}^{+} / \mathrm{GFP}^{+}$cells increased to $73.8 \%$ in the 5 th generation (Fig. 4D-G). The immunocytochemical staining showed that most of the fused cells expressed CD105 (Fig. 5A), whereas, SU3-RFP cells were found to be negative for CD105 (Fig. 5B).

Fused cells display angiogenic properties in vitro. When cultured on Matrigel, the fused cells changed from a cluster of cells to sparse links between cells (day 1) (Fig. 5C) and gradually formed continuous net-like structures (day 3) (Fig. 5D).

Fused cells generate newly formed tumor vessels in vivo. To investigate the ability of fused cells to form endothelial vessels in vivo, the $\mathrm{RFP}^{+} / \mathrm{GFP}^{+}$cells were sorted by FACS and grafted into the Foxn1 ${ }^{\text {nu }}$ mice. After 3-4 weeks, highly vascularized anaplastic tumors were detected; blood vessels were abundant in the H\&E-stained images of the grafted specimens (Fig. 6A and B). A vessel containing red blood cells is indicated by a black arrow in Fig. 6B. Immunohistochemical staining on the adjacent section of the same specimen indicated that $\mathrm{CD} 105^{+}$cells were distributed in the transplanted tumors, particularly on the not fully mature vascular walls (Fig. 6C). Another adjacent section was observed under a fluorescence microscope. We found that the tumor cells co-expressed RFP and GFP; simultaneously fused cells were also involved in vessel wall formation in the xenograft tumor model (Fig. 6D-F).

$\mathrm{RFP}^{+} / \mathrm{GFP}^{+}$blood vessels in dual-color transplanted tumor tissue. We transplanted SU3-RFP cells into the brains of NC-C57BL/6J-GFP nude mice, and the xenograft tumors were examined by fluorescence microscopy. Under fluorescence microscopy, tumor (red) and peritumoral brain tissues (green) were easily distinguished (Fig. 7A). A fraction of tube-like 

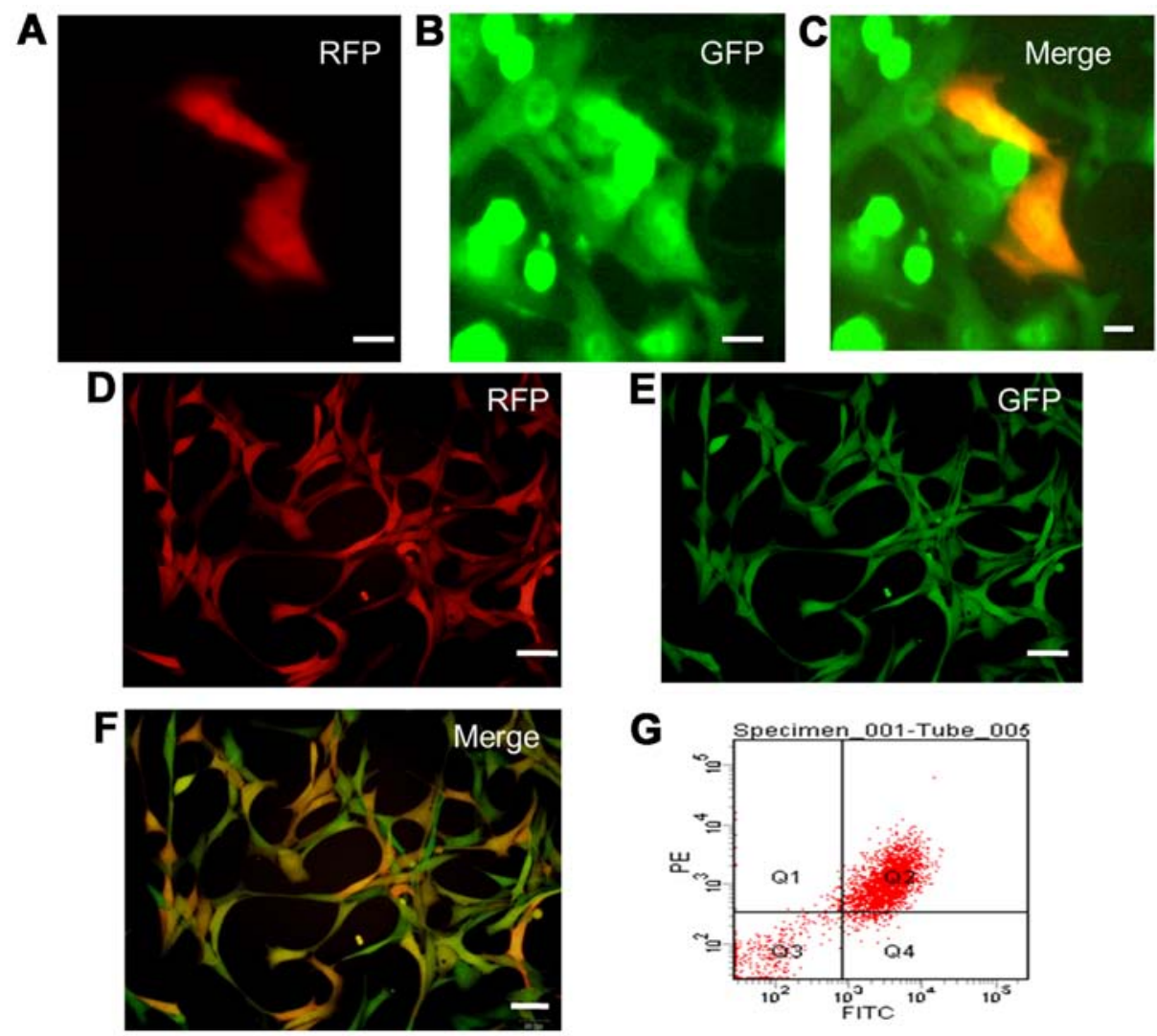

Figure 4. Detection of fused cells by inverted fluorescence microscopy. (A-C) SU3-RFP cells showed red fluorescence and BMSCs showed green fluorescence. Cells co-expressing RFP (A) and RFP (B) were detected in the merged image (C) which showed yellow fluorescence indicating tumor-host cell fusion. (D-G) With the extension of culture and passage, the proportion of fused cells increased. $\mathrm{RFP}^{+} / \mathrm{GFP}^{+}$cells comprised the absolute major proportion and accounted for $73.8 \%$ of the co-cultured cell assembly as detected by flow cytometry (scale bars, $20 \mu \mathrm{m}$ ). BMSCs, bone marrow-derived mesenchymal stem cells.
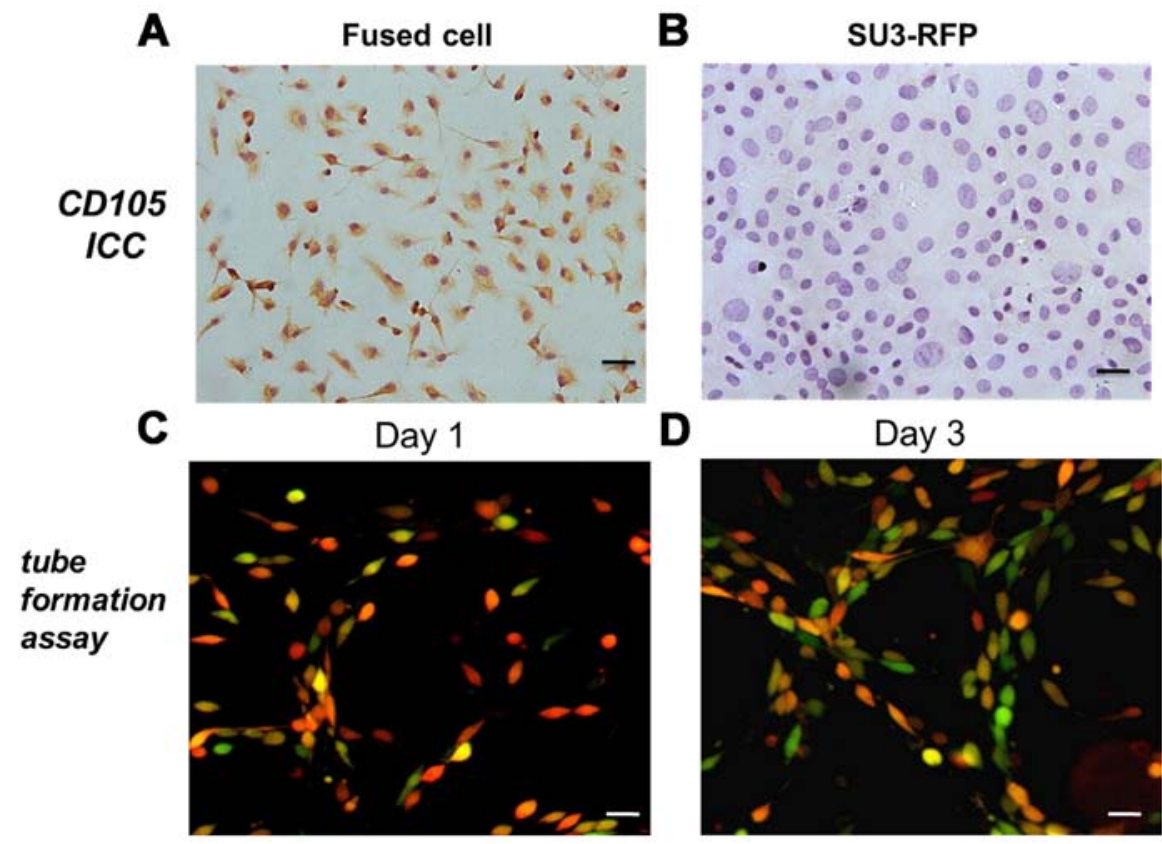

Figure 5. CD105 expression and tube formation ability of the fused cells. (A) CD105 was strongly expressed in the fused cells, while (B) CD105 was found to be absent in the SU3-RFP cells. When cultured on Matrigel, fused cells changed from (C) a cluster of cells to sparse links between cells (day 1), and (D) gradually formed continuous net-like structures (day 3).

structures co-expressing RFP and GFP were detected in the preformed tissue slice (Fig. 7B), suggesting the occurrence of cell fusion in the tumors. Transverse sections of the blood vessels were observed under fluorescence microscopy. Merged 

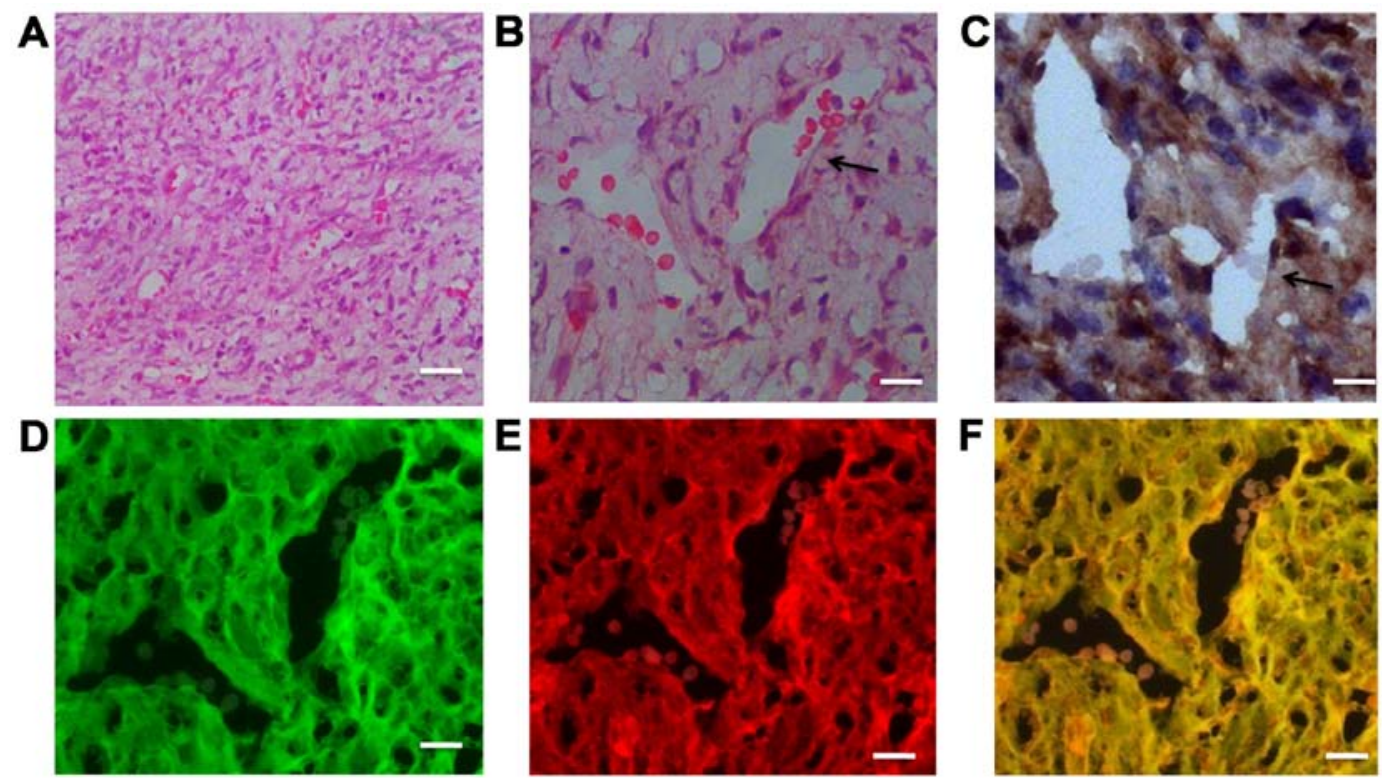

Figure 6. Foxn ${ }^{\text {nu }}$ mice were inoculated with $\mathrm{RFP}^{+} / \mathrm{GFP}^{+}$fused cells. Images of $\mathrm{H} \& \mathrm{E}$ - and $\mathrm{CD} 105$-stained transplanted tumors were from consecutive sections, with a distance of $5 \mu \mathrm{m}$. Adjacent sections were observed by fluorescence microscopy. (A) Microvascular proliferation was detected in a paraffin-embedded H\&E-stained section (magnification, x200). (B) In a magnified view of a H\&E stained section (magnification, $\mathrm{x} 400$ ), a vessel containing red blood cells is indicated by a black arrow. (C) In an adjacent section of the same specimen, immunohistochemical staining for CD105 showed that both tumor cells and vessels were stained positively for CD105. Consecutive sections observed under fluorescence microscopy indicated that the tumor cells and tumor vessels were both positive for (D) RFP and (E) GFP, which were yellow when merged (F). GFP, green fluorescent protein; H\&E, hematoxylin and eosin.

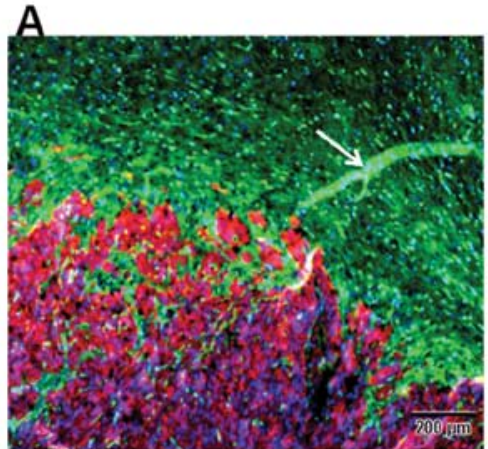

D

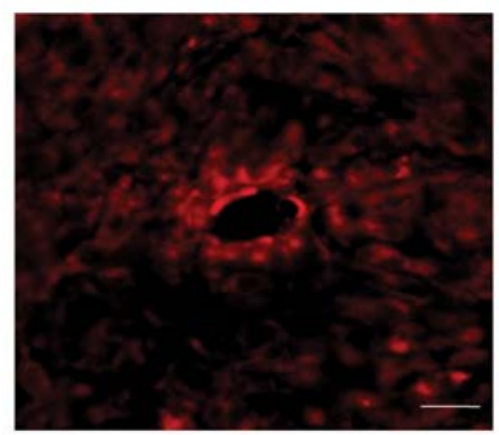

B

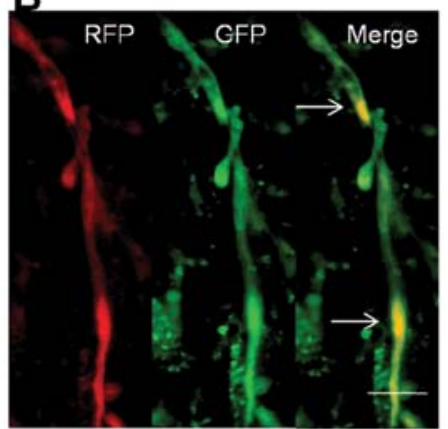

E

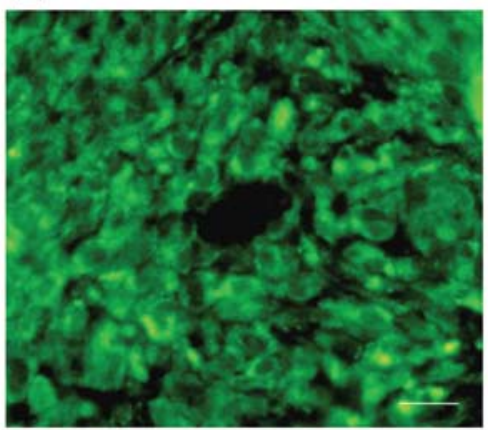

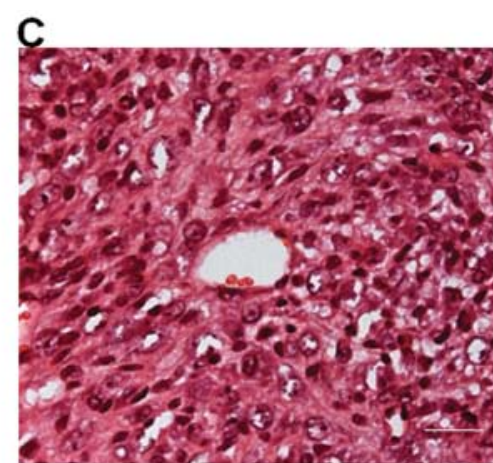

$\mathbf{F}$

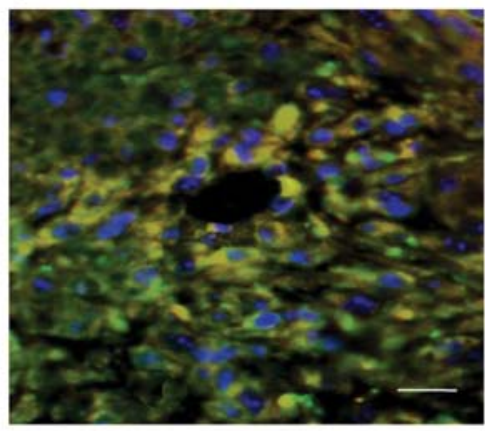

Figure 7. In vivo fluorescence tracing of the tumor tissues and vessels following transplantion of SU3-RFP cells into NC-C57BL/6J-GFP nude mice. (A) Xenograft tumor sections with nuclei stained by DAPI were observed by fluorescence microscopy. Tumor (red) and brain tissue adjacent to the xenograft (green) and host-derived vessels (green, arrow) are shown. (B) A vessel containing $\mathrm{RFP}^{+} / \mathrm{GFP}^{+}$cells is indicated (arrow). (C) Image of $\mathrm{H} \& \mathrm{E}$ stained transverse sectioned blood vessel. (D-F) Adjacent section was observed under fluorescence microscope, most cells on the vessel wall were positive for RFP (D), GFP as well (E), co-expression were shown in (F), outside the lumen, $\mathrm{RFP}^{+} / \mathrm{GFP}^{+}$cells could also be found.

images showed that these $\mathrm{RFP}^{+} / \mathrm{GFP}^{+}$blood vessels came from the host vascular wall where the SU3-RFP cells localized and formed tumor vessels by cell fusion since only SU3 cells successfully transferred with the RFP gene would express red fluorescence, and only the host-derived vessel cells would express green fluorescence (Fig. 7F). 

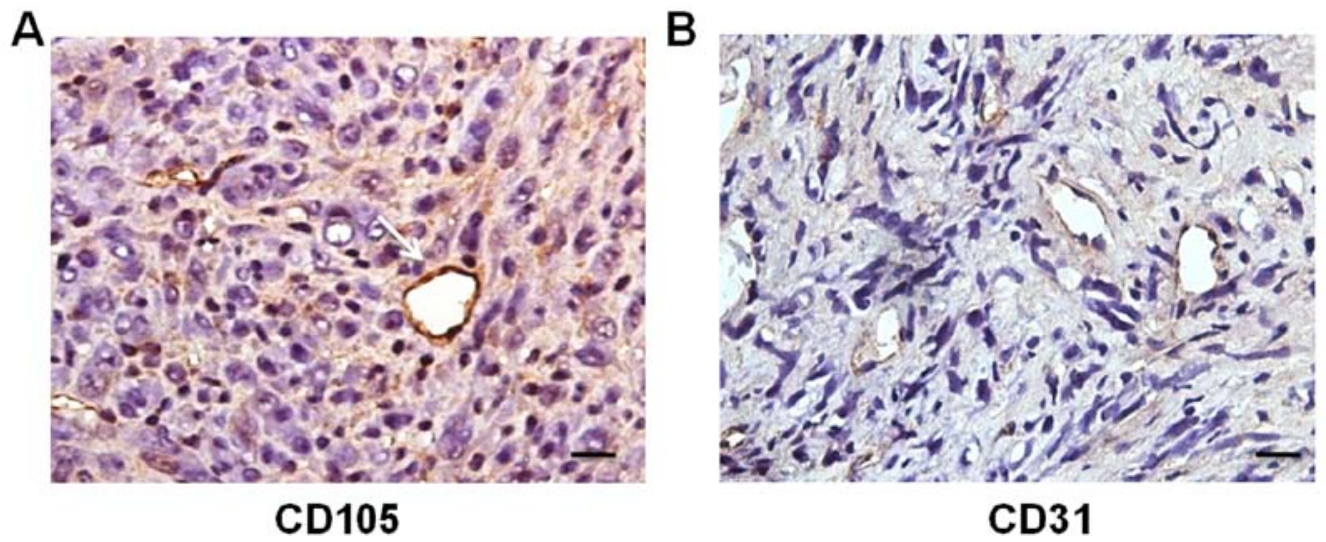

Figure 8. Immunohistochemical staining for $\mathrm{CD} 31$ and $\mathrm{CD} 105$ in a GFP/RFP dual-color tumor specimen. (A) Adjacent section from Fig. 7F. The RFP $/ \mathrm{GFP}^{+}$ vessel is positive for immunohistochemical staining for CD105 (magnification, $\mathrm{x} 400$ ). (B) Most of the tumor vessels are positive for CD31 (magnification, x400). Scare bar, $25 \mu \mathrm{m}$.

Whether tumor cells fused with host BMSCs was not certain, since the host cells were GFP-expressing. Immunohistochemical staining for $\mathrm{CD} 105$ on adjacent sections of the $\mathrm{RFP}^{+} / \mathrm{GFP}^{+}$ vessels was positive (Fig. 8A), indicating that the $\mathrm{CD} 105^{+}$ vessels originated from the fusion of SU3-RFP cells and host BMSCs. Meanwhile, most of the tumor vessels were positive for CD31 (Fig. 8B).

\section{Discussion}

Cancer stem cells are a small fraction of cancer cells that have the potential to drive tumorigenesis. Although cancer stem cell models are controversial for some tumors, glioblastoma stem cells have been reported to give rise to endothelial cells (ECs) $(8,9)$ and pericytes (16). However, some authors pointed out that the discrepancies in this research may be caused by neglecting the function of cell fusion; the cell-fusion-dependent event was mistaken for cancer stem cell plasticity (17). Meanwhile, there is also growing interest in the role of mesenchymal stem cells in tumor angiogenesis. Bone marrow-derived mesenchymal stem cells (BMSCs) are a population of non-hematopoietic stem cells in the bone marrow microenvironment that have the ability to self-renew and multilineage differentiation potential. There is accumulating evidence indicating that BMSCs differentiate into endothelial-like cells $(18,19)$. However, the roles of the interaction between BMSCs and tumor cells in the processes of tumor growth and angiogenesis have not been well studied (20), and have currently generated strong controversy. MSCs were even described as 'double-edged swords' since they have stimulatory (21) or inhibitory (22) effects on tumor angiogenesis and progression. Recently, cells resembling MSCs were able to be isolated from human glioma specimens (23) and glioma xenografts (24). Kim et al (24) found that these cells had similar surface markers to MSCs and were located around vessels, yet they failed to clarify the biological relationship between MSCs and glioma stem cells, and showed weak evidence to prove the absence of gliomagenesis characteristics. It cannot be excluded that BMSC may be recruited and misused by tumor cells, which could be beneficial for tumor angiogenesis, invasion, survival as a consequence of cell fusion (25). To confirm this hypothesis, we used fluorescence tracing in vivo and in vitro to indicate interac- tions between RFP-labeled human tumor cells and GFP-labeled murine BMSCs under direct vision.

In our research, we used short-term cultured mouse BMSCs established in vitro. However, whether the biological characteristics of these cells were suitable for such research is uncertain. The present study indicated that BMSCs were suitable due to their strong colony-forming capacity, stable CD105 and GFP overexpression (Figs. 1 and 2).

Although there are no unique markers for BMSCs, it is generally agreed that human BMSCs are positive for CD105, CD90 and CD73 and negative for CD34, CD45, CD14, CD11b, CD79a, CD19 or HLA-DR surface molecules. In addition, they must be able to differentiate to osteoblasts, adipocytes, and chondroblasts in vitro, as BMSCs have multiple differentiation abilities (26). Yet, these criteria apply only to human MSCs, as for murine MSCs, surface antigen expression is not universally well characterized (27).

In the present study, we found that BMSCs isolated from bone marrow of NC-C57BL/6J-GFP nude mice expressed high levels of CD105 and CD44, yet very low levels of CD45 and CD11b. CD44 glycoproteins are integral cell membrane components widely distributed on various types of cells that function as adhesion molecules of epithelial cells. CD105 (endoglin) is a type I membrane glycoprotein located on cell surfaces and is part of the TGF- $\beta$ receptor complex. Recently, CD105 has been identified as a unique marker of proliferating endothelial cells in vitro and in vivo. It is preferentially expressed in the angiogenic endothelium (28). Since these markers are not specific for BMSCs, they are mainly characterized by their ability to differentiate into multiple mesenchymal lineages. In our study, BMSCs could differentiate into osteoblasts, adipocytes, and chondroblasts in vitro.

Wang et al (9) indicated that $\mathrm{CD} 105^{+}$cells were essentially absent in normal brain. In our research, we did not detect the expression of CD105 in the SU3-RFP cells (Fig. 5B) or in normal murine brain tissues (data not shown). However, we found that CD105 was expressed by BMSCs at a high level. Thus, we confirmed that the $\mathrm{GFP}^{+} / \mathrm{CD} 05^{+}$cells could trace murine BMSCs in the present study.

In the co-culture of SU3-RFP cells and BMSCs, we harvested $\mathrm{RFP}^{+} / \mathrm{GFP}^{+}$cells (Fig. 4), and immunocytochemical 
staining of these cells displayed CD105 expression, confirming the fusion event of glioma stem cells and BMSCs.

In vivo, fused cells have the ability to generate tumor vessels. In addition, $\mathrm{CD}_{105} / \mathrm{RFP}^{+} / \mathrm{GFP}^{+}$vessels were observed in the dual-color orthotopic xenograft glioma specimens (Figs. 7 and 8), perticularly on intratumoral blood vessels. We concluded that the green component in the $\mathrm{RFP}^{+} / \mathrm{GFP}^{+}$ blood vessels was derived from the $\mathrm{CD} 105^{+} \mathrm{BMSC}$ of the GFP mice and hypothesized that this transdifferentiation potential was acquired through cell fusion.

Increasing evidence indicates that inappropriate cell-cell fusion contributes to cancer initiation and progression (29-31). It also contributes to the aneuploidy and the aberrant gene expression patterns associated with malignant cells. Moreover, some scholars believe that fusion of an adult stem cell with a differentiated cell leads to the formation of a cancer stem cell (29).

According to the assumption by Lu and Kang (32), cell fusions mainly take the following forms: stem-differentiated, stem-stem and differentiated-differentiated cells. The fusion between BMSCs and SU3-RFP cells in this experiment was an example of stem-stem cell fusion. In fact, the differentiation direction after cell fusion should be given more attention. Quintana-Bustamante et al (33) and Suzuki et al (34) reported that fused cells possess greater plasticity. In this experiment, the fused cells of BMSCs and SU3-RFP also showed such features. The in vitro test showed that the fused cells had splitting and differentiation potentials; fused cells experienced sequential morphological changes towards the shape of endothelial cells until formation of vessel-like tubes on the Matrigel, while the in vivo test found that the fused cells formed tumor blood vessels and tumor cells. However, the entire process from the beginning of the fusion between the BMSCs and SU3-RFP cells to the driving of neovascularization in vivo was not observed. Rather, we only speculated from the angiogenesis and vasculogenesis theory of Carmeliet and Jain (3) that it belonged to tumor-endothelial cell transdifferentiation. Furthermore, we believed that this transdifferentiation potential was acquired through the cell fusion process itself.

Cell fusion is a nuclear reprogramming technique that involves two or more cell types to form a single identity. However, its molecular mechanism and how fused cells contribute to tumor neovascularization remains unclear. A general requirement for cell fusion is that the two fusing membranes are in close contact, which may be mediated by receptor-ligand interactions (25). Recently, Shinojima et al (35) identified TGF- $\beta$ as a tumor factor that attracts BMSC homing to GSCs via TGF- $\beta$ receptors on BMSCs.

TGF- $\beta$ promotes tumor development and neovascularization by regulating cell proliferation, differentiation and migration. As the TGF- $\beta$ accessory receptor III, CD105 promotes neovascularization by binding with TGF- $\beta 1$ and TGF- $\beta 3$ with high affinity as reported by Fonsatti et al (36) and Warrington et al (37).

The tumor neovascularization in this experiment was realized by the fusion between tumor cells and BMSCs, a process that may be regulated by TGF- $\beta$. A study on the regulatory relationship between CD105 and the TGF- $\beta$ family in the neovascularization of glioma driven by cell fusion is in progress.
Taken together, whether the mechanisms of neovascularization and the origin of tumor endothelial cells are related to the differentiation of tumor stem/progenitor cells or the hyperplasia of host vascular endothelium remains poorly defined. These two paradoxical theories may be unified in the assumption proposed in the present study. That is, tumor cells and host BMSCs become fused to promote tumor neovascularization. There are intricate mechanisms in cell-to-cell as well as in cell-to-ECM cross-talks, which finally guide stem cell fate and behavior. We assumed that cell fusion gives the fused cells a particular potential for proliferation, differentiation and transdifferentiation to form tumor vessels to meet the increased demand for blood supply under a condition of increased tumor cell proliferation.

\section{Acknowledgements}

The present study was supported by grants from the National Natural Science Foundation of China (nos. 81272799, 81172400,81071766 and 81172401 ), a project funded by the priority Academic Program Development of Jiangsu Higher Education Institutions, and the Scientific and Technological Development Plan Project of Suzhou (no. SYSD2012090).

\section{References}

1. Plate KH, Scholz A and Dumont DJ: Tumor angiogenesis and anti-angiogenic therapy in malignant gliomas revisited. Acta Neuropathol 124: 763-775, 2012.

2. Ping YF and Bian XW: Concise review: Contribution of cancer stem cells to neovascularization. Stem Cells 29: 888-894, 2011.

3. Carmeliet P and Jain RK: Molecular mechanisms and clinical applications of angiogenesis. Nature 473: 298-307, 2011.

4. Maniotis AJ, Folberg R, Hess A, Seftor EA, Gardner LM, Pe'er J, Trent JM, Meltzer PS and Hendrix MJ: Vascular channel formation by human melanoma cells in vivo and in vitro: Vasculogenic mimicry. Am J Pathol 155: 739-752, 1999.

5. El Hallani S, Boisselier B, Peglion F, Rousseau A, Colin C, Idbaih A, Marie Y, Mokhtari K, Thomas JL, Eichmann A, et al: A new alternative mechanism in glioblastoma vascularization: Tubular vasculogenic mimicry. Brain 133: 973-982, 2010.

6. Dong J, Zhao Y, Huang Q, Fei X, Diao Y, Shen Y, Xiao H, Zhang T, Lan Q and Gu X: Glioma stem/progenitor cells contribute to neovascularization via transdifferentiation. Stem Cell Rev 7: 141-152, 2011.

7. Zhao Y, Dong J, Huang Q, Lou M, Wang A and Lan Q: Endothelial cell transdifferentiation of human glioma stem progenitor cells in vitro. Brain Res Bull 82: 308-312, 2010.

8. Ricci-Vitiani L, Pallini R, Biffoni M, Todaro M, Invernici G, Cenci T, Maira G, Parati EA, Stassi G, Larocca LM, et al: Tumour vascularization via endothelial differentiation of glioblastoma stem-like cells. Nature 468: 824-828, 2010.

9. Wang R, Chadalavada K, Wilshire J, Kowalik U, Hovinga KE, Geber A, Fligelman B, Leversha M, Brennan C and Tabar V: Glioblastoma stem-like cells give rise to tumour endothelium. Nature 468: 829-833, 2010.

10. Hong IS, Lee HY and Kang KS: Mesenchymal stem cells and cancer: Friends or enemies? Mutat Res 768: 98-106, 2014.

11. Dong J, Zhang Q, Huang Q, Chen H, Shen Y, Fei X, Zhang T, Diao $\mathrm{Y}, \mathrm{Wu} \mathrm{Z}$, Qin Z, et al: Glioma stem cells involved in tumor tissue remodeling in a xenograft model. J Neurosurg 113: 249-260, 2010.

12. Huang Q, Zhang QB, Dong J, Wu YY, Shen YT, Zhao YD, Zhu YD, Diao Y, Wang AD and Lan Q: Glioma stem cells are more aggressive in recurrent tumors with malignant progression than in the primary tumor, and both can be maintained long-term in vitro. BMC Cancer 8: 304, 2008.

13. Dong J, Dai XL, Lu ZH, Fei XF, Chen H, Zhang QB, Zhao YD, Wang ZM, Wang AD, Lan Q, et al: Incubation and application of transgenic green fluorescent nude mice in visualization studies on glioma tissue remodeling. Chin Med J 125: 4349-4354, 2012. 
14. Wu Z, Huang Q, Shao YX, Xue ZM, Dong J, Diao Y, Wang AD and Lan Q: Transplantation of human glioma stem cells in nude mice with green fluorescent protein expression. Zhonghua Yi Xue Za Zhi 88: 2317-2320, 2008 (In Chinese).

15. Liu H, Toh WS, Lu K, MacAry PA, Kemeny DM and Cao T: A subpopulation of mesenchymal stromal cells with high osteogenic potential. J Cell Mol Med 13: 2436-2447, 2009.

16. Cheng L, Huang Z, Zhou W, Wu Q, Donnola S, Liu JK, Fang X, Sloan AE, Mao Y, Lathia JD, et al: Glioblastoma stem cells generate vascular pericytes to support vessel function and tumor growth. Cell 153: 139-152, 2013.

17. Liu AY and Ouyang G: Tumor angiogenesis: A new source of pericytes. Curr Biol 23: R565-R568, 2013.

18. Silva GV, Litovsky S, Assad JA, Sousa AL, Martin BJ, Vela D, Coulter SC, Lin J, Ober J, Vaughn WK, et al: Mesenchymal stem cells differentiate into an endothelial phenotype, enhance vascular density, and improve heart function in a canine chronic ischemia model. Circulation 111: 150-156, 2005.

19. Janeczek Portalska K, Leferink A, Groen N, Fernandes H, Moroni L, van Blitterswijk C and de Boer J: Endothelial differentiation of mesenchymal stromal cells. PLoS One 7: e46842, 2012

20. Huang WH, Chang MC, Tsai KS, Hung MC, Chen HL and Hung SC: Mesenchymal stem cells promote growth and angiogenesis of tumors in mice. Oncogene 32: 4343-4354, 2013.

21. Beckermann BM, Kallifatidis G, Groth A, Frommhold D, Apel A, Mattern J, Salnikov AV, Moldenhauer G, Wagner W, Diehlmann A, et al: VEGF expression by mesenchymal stem cells contributes to angiogenesis in pancreatic carcinoma. Br J Cancer 99: 622-631, 2008.

22. Ho IA, Toh HC, Ng WH, Teo YL, Guo CM, Hui KM and Lam PY: Human bone marrow-derived mesenchymal stem cells suppress human glioma growth through inhibition of angiogenesis. Stem Cells 31: 146-155, 2013.

23. Kim SM, Kang SG, Park NR, Mok HS, Huh YM, Lee SJ, Jeun SS, Hong YK, Park CK and Lang FF: Presence of glioma stroma mesenchymal stem cells in a murine orthotopic glioma model. Childs Nerv Syst 27: 911-922, 2011.

24. Kim YG, Jeon S, Sin GY, Shim JK, Kim BK, Shin HJ, Lee JH, Huh YM, Lee SJ, Kim EH, et al: Existence of glioma stroma mesenchymal stemlike cells in Korean glioma specimens. Childs Nerv Syst 29: 549-563, 2013.

25. Schichor C, Albrecht V, Korte B, Buchner A, Riesenberg R, Mysliwietz J, Paron I, Motaln H, Turnšek TL, Jürchott K, et al: Mesenchymal stem cells and glioma cells form a structural as well as a functional syncytium in vitro. Exp Neurol 234: 208-219, 2012.
26. Dominici M, Le Blanc K, Mueller I, Slaper-Cortenbach I, Marini F, Krause D, Deans R, Keating A, Prockop D and Horwitz E: Minimal criteria for defining multipotent mesenchymal stromal cells. The International Society for Cellular Therapy position statement. Cytotherapy 8: 315-317, 2006.

27. Tropel P, Noël D, Platet N, Legrand P, Benabid AL and Berger F: Isolation and characterisation of mesenchymal stem cells from adult mouse bone marrow. Exp Cell Res 295: 395-406, 2004.

28. Dallas NA, Samuel S, Xia L, Fan F, Gray MJ, Lim SJ and Ellis LM: Endoglin (CD105): A marker of tumor vasculature and potential target for therapy. Clin Cancer Res 14: 1931-1937, 2008.

29. Bjerkvig R, Tysnes BB, Aboody KS, Najbauer J and Terzis A: Opinion: The origin of the cancer stem cell: Current controversies and new insights. Nat Rev Cancer 5: 899-904, 2005.

30. Dittmar T, Nagler C, Schwitalla S, Reith G, Niggemann B and Zänker KS: Recurrence cancer stem cells - made by cell fusion? Med Hypotheses 73: 542-547, 2009.

31. Lu X and Kang Y: Cell fusion as a hidden force in tumor progression. Cancer Res 69: 8536-8539, 2009.

32. Lu X and Kang Y: Cell fusion hypothesis of the cancer stem cell. In: Cell Fusion in Health and Disease. Springer, pp129-140, 2011.

33. Quintana-Bustamante O, Alvarez-Barrientos A, Kofman AV, Fabregat I, Bueren JA, Theise ND and Segovia JC: Hematopoietic mobilization in mice increases the presence of bone marrow-derived hepatocytes via in vivo cell fusion. Hepatology 43: 108-116, 2006.

34. Suzuki K, Sun R, Origuchi M, Kanehira M, Takahata T, Itoh J, Umezawa A, Kijima H, Fukuda S and Saijo Y: Mesenchymal stromal cells promote tumor growth through the enhancement of neovascularization. Mol Med 17: 579-587, 2011.

35. Shinojima N, Hossain A, Takezaki T, Fueyo J, Gumin J, Gao F, Nwajei F, Marini FC, Andreeff M, Kuratsu J, et al: TGF- $\beta$ mediates homing of bone marrow-derived human mesenchymal stem cells to glioma stem cells. Cancer Res 73: 2333-2344, 2013.

36. Fonsatti E, Nicolay HJ, Altomonte M, Covre A and Maio M: Targeting cancer vasculature via endoglin/CD105: A novel antibody-based diagnostic and therapeutic strategy in solid tumours. Cardiovasc Res 86: 12-19, 2010.

37. Warrington K, Hillarby MC, Li C, Letarte M and Kumar S: Functional role of CD105 in TGF-betal signalling in murine and human endothelial cells. Anticancer Res 25: 1851-1864, 2005. 